\title{
Canine Serum Amyloid A (SAA) Measured by Automated Latex Agglutination Turbidimetry Is Useful for Routine Sensitive and Specific Detection of Systemic Inflammation in a General Clinical Setting
}

\author{
Michelle B. CHRISTENSEN ${ }^{1) *}$, Rebecca LANGHORN ${ }^{1)}$, Amelia GODDARD ${ }^{2)}$, Eva B. ANDREASEN ${ }^{1)}$, \\ Elena MOLDAL ${ }^{3)}$, Asta TVARIJONAVICIUTE ${ }^{4)}$, Jolle KIRPENTEIJN ${ }^{5}$, Sabrina JAKOBSEN ${ }^{1}$, Frida PERSSON ${ }^{1)}$ and \\ Mads KJELGAARD-HANSEN ${ }^{1)}$ \\ ${ }^{1)}$ Department of Veterinary Clinical and Animal Sciences, University of Copenhagen, Ground Floor, 3 Groennegaardsvej, 1870 \\ Frederiksberg C, Denmark \\ ${ }^{2)}$ Department of Companion Animal Clinical Studies, University of Pretoria, Private Bag X04, Onderstepoort, 0110, Pretoria, South Africa \\ ${ }^{3}$ Department of Companion Animal Sciences, Norwegian School of Veterinary Science, P.O. Box 8146 Dep. N-0033 Oslo, Norway \\ ${ }^{4)}$ Department of Animal Medicine and Surgery, The Veterinary Faculty, Campus de Espinardo, University of Murcia, s/n 30100 Espinardo, \\ Murcia, Spain \\ ${ }^{5}$ Department of Clinical Sciences of Companion Animals, Faculty of Veterinary Medicine, University of Utrecht, Yalelaan 108, 3384 CM \\ Utrecht, Netherlands
}

(Received 11 September 2012/Accepted 14 November 2012/Published online in J-STAGE 28 November 2012)

\begin{abstract}
Canine serum amyloid A (SAA) is a useful diagnostic marker of systemic inflammation. A latex agglutination turbidimetric immunoassay (LAT) was validated for automated measurements. The aim of the study was to evaluate the clinical applicability of SAA measured by the LAT. SAA was measured in 7 groups of dogs with and without systemic inflammation ( $\mathrm{n}=247$ ). Overlap performance was investigated. Diagnostic performance was compared to body temperature and leukocyte markers. Clinical decision limits for SAA were estimated. In dogs with neurological, neoplastic or gastrointestinal disorders $(n=143)$, it was investigated whether a higher proportion of SAA positive dogs could be detected in cases of complications with risk of systemic inflammation. Significantly higher concentrations of SAA were measured in dogs with (range $[48.75 ; 5,032 \mathrm{mg} / l]$ ), compared to dogs without systemic inflammation $[0 ; 56.4 \mathrm{mg} / l]$. SAA was a more sensitive and specific marker of systemic inflammation (area under the receiver-operating characteristic curve (AUC) 1.00), compared to body temperature (0.6) and segmented neutrophils (best performing leukocyte marker, 0.84). A clinical decision limit of $56.4 \mathrm{mg} / \mathrm{l}$ was established giving close to perfect discrimination between dogs with and without systemic inflammation. Higher proportions of SAApositive dogs were observed in dogs with neurological, neoplastic and gastrointestinal disorders with complications known to increase risk of systemic inflammation, compared to uncomplicated cases. The automated LAT makes SAA applicable as a relevant diagnostic marker of systemic inflammation in dogs for routine random-access real-time use in a general clinical setting.

KEY WORDS: canine, inflammation, veterinary clinical pathology.
\end{abstract}

doi: 10.1292/jvms.12-0404; J. Vet. Med. Sci. 75(4): 459-466, 2013

The acute phase response (APR) is an unspecific systemic reaction, which follows various stimuli such as infection or trauma, causing systemic inflammation and disrupting homeostasis $[3,21]$. During the APR, the concentrations of acute phase proteins (APP) are altered [21]. Serum amyloid A (SAA) is a major positive APP in dogs, and marked increases in concentrations are consequently observed during the APR [3]. Studies in several species have shown that SAA is a more sensitive marker of systemic inflammation than traditionally used parameters such as body temperature, leukocyte and neutrophil counts [12, 19], and SAA can be used as a diagnostic marker of systemic inflammation in several species, including dogs $[1,4,8,21]$. The commercial availability of diagnostic assays has, however, been limited to

*Correspondence to: Christensen, M. B., Department of Veterinary Clinical and Animal Sciences, University of Copenhagen, Ground Floor, 3 Groennegaardsvej, 1870 Frederiksberg C, Denmark. e-mail:mic@sund.ku.dk

C2013 The Japanese Society of Veterinary Science resource and time consuming methods, and so far measurements of canine SAA have not been implemented in routine veterinary clinical biochemistry [3]. The initial evaluations of an automated latex agglutination turbidimetric immunoassay (LAT, EIKEN, Tokyo, Japan), based on human monoclonal antibodies, have shown acceptable analytical and overlap performance [7], making routine diagnostic measurements of canine SAA possible. Previous studies of canine SAA have focused on specific disorders such as meningitis [18], neoplasia [23] or pyometra [8], but more knowledge of the diagnostic performance of SAA for a wider spectrum of disorders will be needed before SAA can be implemented as a routine marker of systemic inflammation for use in a general clinical setting [17].

Initially, 3 aspects were investigated in the present study: First, the overlap performance of SAA was investigated thoroughly, based on the hypothesis that SAA can be used to differentiate dogs with systemic inflammation from diseased dogs with non-inflammatory disorders and clinically healthy dogs. Second, the diagnostic performance was compared to the traditional inflammatory parameters, body temperature, 
Table 1. Overview of the composition of groups and origin of dogs included in the study

\begin{tabular}{|c|c|c|}
\hline Group & $\mathrm{n}$ & Composition of groups \\
\hline Clinically healthy dogs* & 76 & Health screening $(n=29)^{a)}$, healthy controls in previous studies $\left.(n=47)^{a-d}\right)$ \\
\hline Diseased dogs without systemic inflammation* & 35 & See table 2 for details ${ }^{\text {a) }}$ \\
\hline Systemic inflammation induced by aseptic elective surgery & 26 & $\begin{array}{l}\left.\text { Ovariohysterectomy or ovariectomy }{ }^{b}\right)(n=21) \text {, surgical castration of male } \\
\left.\operatorname{dog}^{e}\right)(n=5)\end{array}$ \\
\hline Systemic inflammation caused by accidental trauma & 20 & $\begin{array}{l}\text { Dogs hospitalized for treatment after accidental traumas (e.g. car accidents } \\
\text { or dog bites) })^{a, c, f)}\end{array}$ \\
\hline Systemic inflammation caused by snake envenomation & 45 & $\begin{array}{l}\left.\left.\text { Envenomation by European viper }(n=25)^{d}\right) \text {, African puff adder }(n=8)^{g}\right) \text {, } \\
\text { snouted cobra }(n=10)^{g)} \text { or Mozambique spitting cobra }(n=2)^{g)}\end{array}$ \\
\hline Systemic inflammation due to various miscellaneous causes & 35 & See table 2 for details ${ }^{\mathrm{a}, \mathrm{c})}$ \\
\hline Dogs with aspiration pneumonia & 10 & Aspiration during hospitalization, confirmed by radiographic evaluations ${ }^{\mathrm{c}}$ ) \\
\hline
\end{tabular}

Included dogs were presented at: a) Department of Veterinary Clinical and Animal Sciences, University of Copenhagen, Denmark (2010-2011); b) Department of Clinical Sciences of Companion Animals, University of Utrecht, Netherlands [23]; c) Department of Clinical Studies, School of Veterinary Medicine, University of Pennsylvania, U.S.A. (2011); d) Blue Star Animal Hospital, Gothenburg, Sweden, during (2011); e) Department of Animal Medicine and Surgery, University of Murcia, Spain [26]; f) San Marco Veterinary Clinic, Padova, Italy (2011); g) Department of Companion Animal Clinical Studies, University of Pretoria, South Africa (2010-2011). *Dogs with no expected systemic inflammation.

leukocyte- and neutrophil counts, based on the null-hypothesis that the diagnostic accuracy of SAA to detect systemic inflammation equals that of the traditionally used inflammatory parameters. Third, an optimized clinical decision limit of SAA measured by LAT was estimated, based on the hypothesis that a clinical decision limit for the SAA LAT can be defined to detect the presence of systemic inflammation with clinically relevant diagnostic accuracy.

In some clinical groups of canine patients with inflammation, systemic involvement is not necessarily present, but of clinical importance. Optimally, a marker of systemic inflammation has the ability to differentiate such patients in order to provide valuable information in a general clinical setting. Consequently, dogs with neoplastic, neurologic and gastrointestinal disorders were included in the final part of the study to represent patients not necessarily systemically affected, but where certain complications are known to increase risk of systemic affection. Thus, detection of systemic inflammation can be of diagnostic and prognostic importance for patients with neurological and neoplastic disorders $[1,5,15,23]$. Consequently, we investigated whether diagnostic measurements of SAA can help to identify these patients, based on the hypothesis that higher proportions of SAA positive dogs will be observed in groups of dogs with inflammatory neurological disorders compared to groups of dogs with non-inflammatory neurological disorders and in dogs with complicated neoplastic disorders compared to dogs with non-complicated neoplastic disorders.

Most cases of gastrointestinal disorders in canine medicine are acute, nonfatal and self-limiting, and they require only symptomatic support, while hemorrhagic diarrhoea and gastrointestinal foreign bodies are two disorders known to increase risk of systemic affection requiring more detailed investigations and more comprehensive therapy $[9,11]$. The objective of the last part of the study was, therefore, to explore whether SAA could possibly be useful in the differentiation of these two groups of patients based on the hypothesis that higher proportions of SAA positive dogs will be observed among dogs with complicated (hemorrhagic diarrhoea or a gastrointestinal foreign body) compared to dogs with self-limiting gastrointestinal symptoms.

\section{MATERIALS AND METHODS}

Serum was obtained by venipuncture of each of the dogs included in the study and stored in plastic vials at -20 to $-80^{\circ} \mathrm{C}$ for a maximum of 5 years. Only serum remaining after other relevant analyses of importance for diagnostic work-up or previous scientific studies [20,26] was used in the present study, and this approach was approved by the local ethical committee, Department of Veterinary Clinical and Animal Sciences, University of Copenhagen, Denmark. The samples were shipped on dry ice (except for the samples obtained after castration and accidental trauma which were shipped on wet ice for maximally $13 \mathrm{hr}$ ) for analysis at the Central Laboratory, Department of Veterinary Clinical and Animal Sciences, University of Copenhagen, Denmark, allowed to thaw to room temperature on the day of analysis, and thoroughly mixed prior to measurements.

SAA in dogs with and without systemic inflammation: Sera from 247 dogs with a mean age of 5.3 years (range, 0.5 years; 14 years) were included in the first part of the study for measurements of SAA in dogs with and without systemic inflammation. Information about leukocyte, segmented neutrophil and band neutrophil counts from the same day as the sampling of serum was available in 173 cases, and information about body temperature at admission was available in 143 of the dogs. $82 \%$ were purebred dogs of 67 different breeds, $48 \%$ of the dogs were males and $52 \%$ were females.

As summarized in Table 1, 7 groups of dogs were established: clinically healthy dogs (client-owned dogs with no signs of clinical illness in history, clinical examination or hematologic and biochemical evaluations), diseased dogs without systemic inflammation (details in Table 2), dogs with systemic inflammation induced by aseptic elective surgery approximately $24 \mathrm{hr}$ prior to serum sampling [20, 
Table 2. Overview of diagnoses of the 70 dogs included with various disorders

\begin{tabular}{lclc}
\hline Various disorders with & $\mathrm{n}$ & Various disorders without & $\mathrm{n}$ \\
systemic inflammation & 35 & systemic inflammation & 35 \\
\hline Polyarthritis & 5 & Hepatic/urogenital disorders & 6 \\
Peritonitis & 7 & Respiratory/cardiovascular & 5 \\
Pyometra & 4 & Orthopedic & 5 \\
Pancreatitis & 3 & Cystitis/anal sacculitis & 3 \\
IMHA/ IMTP & 5 & Dental calculi & 5 \\
Infection & 2 & Dermatologic & 5 \\
Various, inflammatory & 9 & Various, noninflammatory & 6
\end{tabular}

a) Dogs with obvious systemic inflammation e.g. severe stomatitis, allergic reactions, discospondylitis, post-operative abscesses, or pneumonia. b) Dogs suffering from endocrine problems e.g. cushing's disease or disorders included in more than one of the groups mentioned above, e.g. arthrosis and myxomatous mitral valve disorder. Abbreviations: IMHA=Immune mediated hemolytic anemia, IMTP=Immune mediated thrombocytopenia.

26], dogs with systemic inflammation caused by accidental trauma approximately $12-24 \mathrm{hr}$ prior to serum sampling, dogs with systemic inflammation caused by snake envenomation (hospitalized approximately $24 \mathrm{hr}$ prior to serum sampling), dogs with systemic inflammation due to various miscellaneous causes (details in Table 2) and dogs which developed aspiration pneumonia approximately $24 \mathrm{hr}$ prior to serum sampling. While most groups were relatively homogenous in the composition, the dogs with various disorders not accompanied by systematic inflammation, and of dogs with various miscellaneous inflammatory disorders, constituted two heterogeneous groups (Table 2). General clinical examinations were performed in these dogs, and basic hematologic and biochemical profiles were analyzed [14]. Additional diagnostic tests were performed at the discretion of the attending clinicians, including urinalysis, radiography, ultrasonography, cytology, histopathology, endocrine testing or analysis for pancreatic lipases, and conclusions about the diagnoses were based on written information from the attending clinicians.

SAA was measured with LAT (SAA-1, EIKEN Chemical Co., Tokyo, Japan) [7] using an automated clinical chemical analyser (Advia 1800, Siemens, Munich, Germany). Duplicate measurements were performed, and mean concentrations of SAA were used for further statistical analyses. Leukocyte and neutrophil counts were determined using an automated hematology analyzer (Advia 2120, Siemens, Munich, Germany), and all findings were verified by manual microscopic evaluation of blood smears.

Measured concentrations of SAA in the 7 groups of dogs were visualized graphically in a scatter plot using a logarithmic scale, and medians were calculated using routine descriptive statistical procedures. Kruskal-Wallis test was used to test for significant differences in median SAA concentrations between groups of dogs, and Dunn's multiple comparison tests were used for further characterization of observed differences. Significance level was set to $P<0.05$.

The groups were subsequently divided in two units, depending on whether systemic inflammation was part of the disease process or not (Table 1). The diagnostic performance of SAA as a marker of systemic inflammation was subsequently compared to the diagnostic performance of leukocyte count, segmented and band neutrophil counts, and body temperature respectively, by assessment of the area under the curve (AUC) obtained by receiver operating characteristic (ROC) analyses [10]. The parameters were considered to have diagnostic potential for the detection of systemic inflammation if the AUC was found to be above 0.5 [10]. In order to be classified as an efficient marker of systemic inflammation, the $95 \%$ CI of the AUC had to exceed 0.8 . The diagnostic ability of different markers was deemed discrete, if no overlap was observed for the $95 \%$ CI of the respective AUC's.

The ROC analysis was used to identify relevant clinical decision limits [10], defined as the concentrations leading to a diagnostic sensitivity of $100 \%$, a diagnostic specificity of $100 \%$ and the concentration at the maximum differential positive rate $[\operatorname{maxDPR}$, where $\mathrm{DPR}=$ sensitivity $-(1-$ specificity)] for situations where sensitivity or specificity is favoured or equally weighed, respectively. The most general applicable clinical decision limit was defined as the concentration at maxDPR and therefore used for further analyses in the subsequent part of the study.

SAA measurements in dogs without systemic inflammation (clinically healthy dogs and diseased dogs without systemic inflammation) were compared to each of the 5 groups of patients with systemic inflammation separately, to investigate whether the optimal clinical decision limit differed between different types of induction of systemic inflammation. Relevant clinical decision limits in the individual groups were estimated based on the diagnostic sensitivity, diagnostic specificity and maxDPR as described above.

SAA in dogs with diseases including varying degrees of systemic inflammation: Serum from 143 dogs, suffering from neurological, neoplastic or gastrointestinal disorders, with a mean age of 6.3 years (range, [1 year; 14 years]) was included in this part of the study. Eighty-five percent were purebred dogs consisting of 47 different breeds, $43 \%$ were males and $57 \%$ were females. The dogs included in this part of the study were divided into groups based on the type of neurological, neoplastic or gastrointestinal disorder. The composition of the groups is outlined in Table 3.

Dot plots were used for visualization of SAA measurements in dogs suffering from neurological, neoplastic and gastrointestinal disorders. The dogs were further divided into groups based on whether SAA measurements resulted in concentrations above or below the most optimal clinical decision limit calculated in the first part of the study. Chi-square tests were used to test for differences between proportions. The proportions were significantly different, if the $95 \%$ CI of the difference did not include zero, and thus $P<0.05$.

\section{RESULTS}

SAA in dogs with and without systemic inflammation: Diagnostic measurement of canine SAA by the LAT was 
Table 3. Groups of dogs with varying degrees of systemic inflammation

\begin{tabular}{|c|c|c|}
\hline Group & $\mathrm{n}$ & Composition \\
\hline Neurological disorders ${ }^{\mathrm{a}, \mathrm{b}}$ ) & 29 & $\begin{array}{l}\text { Inflammation detected in CSF or by MRI }(n=10) \\
\text { No inflammation detected }(n=19)\end{array}$ \\
\hline Neoplastic disorders ${ }^{\mathrm{a}, \mathrm{b}}$ ) & 85 & $\begin{array}{l}\text { Systemically affected (e.g. metastases, cachexia, DIC, } n=22) \\
\text { Benign neoplasia, no systemic affection }(n=18) \\
\text { Mastocytoma, no systemic affection }(n=7) \\
\text { Malignant neoplasia, no systemic affection }(n=38)\end{array}$ \\
\hline Gastrointestinal disorders ${ }^{\mathrm{a}, \mathrm{b}}$ ) & 29 & $\begin{array}{l}\text { Uncomplicated, self-limiting symptoms }(\mathrm{n}=14) \\
\text { Hemorrhagic gastroenteritis }(\mathrm{n}=12) \\
\text { Gastrointestinal foreign body }(\mathrm{n}=3)\end{array}$ \\
\hline
\end{tabular}

Included dogs were presented at: a) Department of Veterinary Clinical and Animal Sciences, University of Copenhagen, Denmark (2010-2011, n=136); b) Department of Clinical Studies, School of Veterinary Medicine, University of Pennsylvania, U.S.A. (2011, n=7). CSF: Laboratorial analysis of cerebrospinal fluid. DIC: Disseminated intravascular coagulation. MRI: Magnetic resonance imaging.

demonstrated to be clinically useful for detecting systemic inflammation with a significant overlap performance, a higher sensitivity compared to traditional inflammatory markers and a clear clinical decision limit.

Significant higher concentrations of SAA were measured $(P<0.01$, Kruskal-Wallis test, Dunn's multiple comparison test) in dogs $24 \mathrm{hr}$ post-surgery, $12-24 \mathrm{hr}$ post-trauma, 24 $\mathrm{hr}$ post-presentation following snake envenomation, in dogs with systemic inflammation of various causes and in dogs with aspiration pneumonia secondary to other clinical conditions compared to SAA measured in dogs with clinical conditions without systemic inflammation and in clinically healthy dogs, respectively (Fig. 1).

All the inflammatory markers investigated in the study were confirmed to have significant discriminative ability with an AUC above 0.5; SAA, leukocyte count, segmented and band neutrophil counts and body temperature are consequently all useful as diagnostic markers of systemic inflammation (Table 4). However, only SAA qualified as an efficient marker of systemic inflammation with an AUC significantly greater than 0.8 (Table 4). Further, SAA was demonstrated to be more efficient for the detection of systemic inflammation when compared to leukocytes, segmented and band neutrophils and body temperature, respectively, as the $95 \% \mathrm{CI}$ of the AUC of SAA exceeded the $95 \%$ CIs of the AUCs of the other markers without overlap (Table 4).

A relevant clinical decision limit for SAA was observed to be between 47.8 and $56.4 \mathrm{mg} / \mathrm{l}$ depending on whether the diagnostic sensitivity, the diagnostic specificity or the maxDPR was prioritized (Table 5), and the most optimal clinical decision limit varied within the same interval between different sources of systemic inflammation (Table 6). In the present study, $56.4 \mathrm{mg} / \mathrm{l}$ was chosen as the most optimal clinical decision limit used for further analyses, corresponding to the maxDPR in Table 5. With the clinical decision limit set at $56.4 \mathrm{mg} / l$, a maximal diagnostic specificity is obtained without compromising the diagnostic sensitivity, which will remain high: 97.8 (95\% confidence interval, [93.2\%; 99.4\%]). In fact, the sensitivity was demonstrated to be $100 \%$ in 2 of the 5 groups of dogs with systemic inflammation (Table 6).

SAA in dogs with diseases including varying degrees of

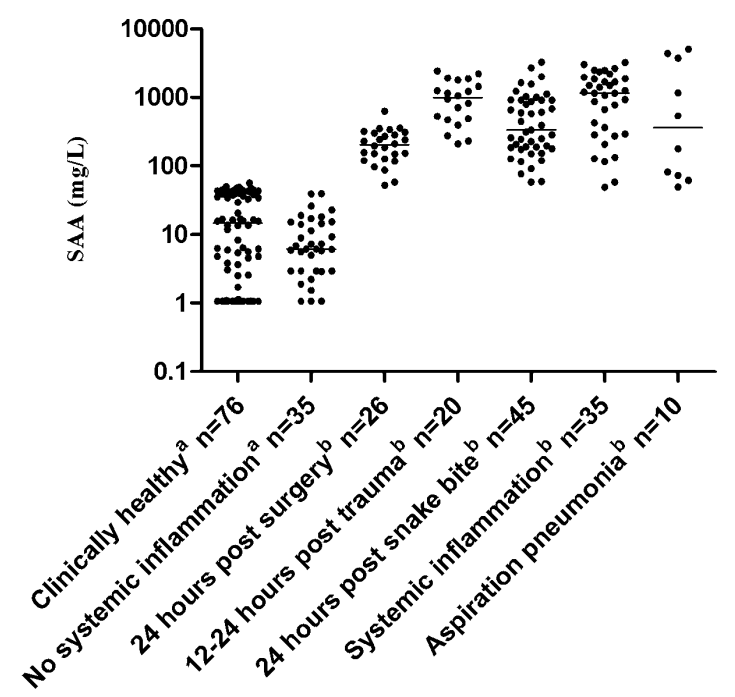

Fig. 1. Scatter plot of serum amyloid A(SAA) measured by a diagnostic latex agglutination turbidimetric immunoassay (LAT) in 7 groups of dogs using a logarithmic scale. Solid lines: Median SAA concentrations. Measurements below detection limit were plotted as $1.06 \mathrm{mg} / l$ which is the previously calculated detection limit of the assay [7]. Groups with different superscript letters are significantly different $(P<0.01$, Kruskal-Wallis and Dunn's multiple comparison test).

systemic inflammation: Concentrations of SAA exceeding the clinical decision limit were detected in 44 of 206 dogs with neurological, neoplastic and gastrointestinal disorders occasionally known to be accompanied by varying degrees of systemic inflammation (Table 7). Increased concentrations of SAA were observed in $60 \%$ ( 6 out of 10 ) of the dogs with neurological disorders and inflammation detected by cytological findings in CSF and/or findings on MRI, while no neurological patients without inflammatory findings had increased levels of SAA. However, four dogs had SAA levels below the clinical decision limit despite of the fact that signs of inflammation were detected. 
Table 4. Receiver operating characteristic curve analysis of SAA and other inflammatory markers

\begin{tabular}{lccccc}
\hline & SAA & $\begin{array}{c}\text { Leukocyte } \\
\text { count }\end{array}$ & $\begin{array}{c}\text { Segmented } \\
\text { neutrophils }\end{array}$ & $\begin{array}{c}\text { Band } \\
\text { neutrophils }\end{array}$ & $\begin{array}{c}\text { Body } \\
\text { temperature }\end{array}$ \\
\hline $\mathrm{n}$ (systemic inflammation) & 136 & 104 & 104 & 104 & 98 \\
$\mathrm{n}$ (no systemic inflammation) & 111 & 69 & 69 & 69 & 45 \\
AUC & 1.00 & 0.80 & 0.84 & 0.65 & 0.60 \\
{$[95 \%$ CI of AUC] } & {$[0.98 ; 1.0]$} & {$[0.73 ; 0.86]$} & {$[0.78 ; 0.89]$} & {$[0.57 ; 0.72]$} & {$[0.52 ; 0.68]$} \\
\hline
\end{tabular}

$\mathrm{AUC}=$ Area under the curve, $\mathrm{CI}=$ Confidence Interval, $\mathrm{SAA}=$ serum amyloid $\mathrm{A}$.

Table 5. Background for estimating a clinical decision limit of serum amyloid A

\begin{tabular}{lccccc}
\hline Clinical decision limit & $\begin{array}{c}\text { Se } \\
(\%)\end{array}$ & $\begin{array}{c}95 \% \text { CI } \\
(\%)\end{array}$ & $\begin{array}{c}\text { Sp } \\
(\%)\end{array}$ & $\begin{array}{c}95 \% \text { CI } \\
(\%)\end{array}$ & $\begin{array}{c}\text { DPR } \\
(\%)\end{array}$ \\
\hline$>47.75 \mathrm{mg} / l$ & $100^{\mathrm{b})}$ & {$[96.6 ; 100]$} & 97.3 & {$[91.7 ; 99.3]$} & 97.3 \\
$>48.75 \mathrm{mg} / l$ & 99.3 & {$[95.4 ; 100]$} & 97.3 & {$[91.7 ; 99.3]$} & 96.6 \\
$>48.85 \mathrm{mg} / l$ & 99.3 & {$[95.4 ; 100]$} & 98.2 & {$[93.0 ; 99.7]$} & 97.5 \\
$>49.55 \mathrm{mg} / l$ & 98.5 & {$[94.2 ; 99.7]$} & 98.2 & {$[93.0 ; 99.7]$} & 96.7 \\
$>50 \mathrm{mg} / l$ & 98.5 & {$[94.2 ; 99.7]$} & 99.1 & {$[94.4 ; 100]$} & 97.6 \\
$>52.3 \mathrm{mg} / l$ & 97.8 & {$[93.2 ; 99.4]$} & 99.1 & {$[94.4 ; 100]$} & 96.9 \\
$>56.4 \mathrm{mg} / l$ & 97.8 & {$[93.2 ; 99.4]$} & $100^{\mathrm{b})}$ & {$[95.8 ; 100]$} & $97.8^{\mathrm{b})}$ \\
\hline
\end{tabular}

a) Receiver operating characteristic curve analysis was used to calculate possible clinical decision limits for SAA measured by latex agglutination turbidimetry. b) The maximal $\mathrm{Se}, \mathrm{Sp}$, and DPR. CI= confidence interval, $\mathrm{Se}=$ sensitivity, $\mathrm{Sp}=$ specificity, $\mathrm{DPR}=$ the maximal differential positive rate $=\mathrm{Se}-(1-\mathrm{Sp}), \mathrm{SAA}=$ serum amyloid $\mathrm{A}$.

Table 6. Relevant clinical decision limits for serum amyloid a in groups of dogs with systemic inflammation

\begin{tabular}{llccccc}
\hline Group & $\begin{array}{c}\text { SAA clinical } \\
\text { decision limit }^{\mathrm{a})}\end{array}$ & $\begin{array}{c}\mathrm{Se} \\
(\%)\end{array}$ & $\begin{array}{c}95 \% \mathrm{CI} \\
(\%)\end{array}$ & $\begin{array}{c}\mathrm{Sp} \\
(\%)\end{array}$ & $\begin{array}{c}95 \% \mathrm{CI} \\
(\%)\end{array}$ & $\begin{array}{c}\text { DPR } \\
(\%)\end{array}$ \\
\hline $24 \mathrm{hr}$ post surgery & $>48.85 \mathrm{mg} / l$ & $100^{\mathrm{b})}$ & {$[84.0 ; 100]$} & 98.2 & {$[93.0 ; 99.7]$} & 98.2 \\
& $>50 \mathrm{mg} / l$ & $100^{\mathrm{b})}$ & {$[84 ; 100]$} & 99.0 & {$[94.0 ; 100]$} & $99.1^{\mathrm{b})}$ \\
& $>56.4 \mathrm{mg} / l$ & 96.2 & {$[78.4 ; 99.8]$} & $100^{\mathrm{b})}$ & {$[95.8 ; 100]$} & 96.2 \\
\multirow{2}{*}{$12-24$ hr post trauma } & $>48.85 \mathrm{mg} / l$ & $100^{\mathrm{b})}$ & {$[80 ; 100]$} & 98.2 & {$[93.0 ; 99.7]$} & 98.2 \\
& $>56.4 \mathrm{mg} / l$ & $100^{\mathrm{b})}$ & {$[80 ; 100]$} & $100^{\mathrm{b})}$ & {$[95.8 ; 100]$} & $100^{\mathrm{b})}$ \\
24 hr post snake-envenomation & $>56.4 \mathrm{mg} / l$ & $100^{\mathrm{b})}$ & {$[90.2 ; 100]$} & $100^{\mathrm{b})}$ & {$[95.8 ; 100]$} & $100^{\mathrm{b})}$ \\
Systemic inflammation & $>47.75 \mathrm{mg} / l$ & $100^{\mathrm{b})}$ & {$[87.7 ; 100]$} & 97.3 & {$[91.7 ; 99.3]$} & $97.3^{\mathrm{b})}$ \\
& $>56.4 \mathrm{mg} / l$ & 97.1 & {$[83.4 ; 99.9]$} & $100^{\mathrm{b})}$ & {$[95.8 ; 100]$} & 97.1 \\
Aspiration pneumonia & $>48.85 \mathrm{mg} / l$ & $100^{\mathrm{b})}$ & {$[65.5 ; 100]$} & 98.2 & {$[93.0 ; 99.7]$} & $98.2^{\mathrm{b})}$ \\
& $>56.4 \mathrm{mg} / l$ & 90 & {$[54.1 ; 99.5]$} & $100^{\mathrm{b})}$ & {$[95.8 ; 100]$} & 90 \\
\hline
\end{tabular}

$\mathrm{SAA}=$ serum amyloid $\mathrm{A}, \mathrm{Se}=$ diagnostic sensitivity, $\mathrm{Sp}=$ diagnostic specificity, $\mathrm{hr}=$ hours after stimulation, $\mathrm{CI}=95 \%$ confidence interval, $\mathrm{DPR}=$ the differential positive rate $=\mathrm{Se}-(1-\mathrm{Sp})$. a) Clinical decision limits were estimated by receiver operating characteristic curve analysis as the concentration with maximal Se, Sp, or DPR. b) The maximal Se, Sp and DPR in each group.

Table 7. SAA in dogs with neurological, neoplastic or gastrointestinal disorders

\begin{tabular}{llcccc}
\hline & $\mathrm{n}$ & $\begin{array}{c}\text { SAA median } \\
(\mathrm{mg} / l)\end{array}$ & $\begin{array}{c}<56.4 \mathrm{mg} / l^{\text {a }} \\
(\%)\end{array}$ & $\begin{array}{c}>56.4 \mathrm{mg} / l^{\text {a }} \\
(\%)\end{array}$ \\
\hline Neurological & Inflammation & 10 & 325 & $4(40)$ & $6(60)$ \\
& No inflammation & 19 & 5.4 & $19(100)$ & $0(0)$ \\
Neoplastic & Complicated $^{b}$ & 22 & 81.8 & $7(32)$ & $15(68)$ \\
& Uncomplicated & 63 & 0 & $61(97)$ & $2(3)$ \\
& Benign & 18 & 0 & $18(100)$ & $0(0)$ \\
& Mastocytoma & 7 & 0 & $7(100)$ & $0(0)$ \\
& Malignant & 38 & 0.3 & $36(95)$ & $2(5)$ \\
Gastrointestinal & Complicated $)$ & 15 & 269 & $2(13)$ & $13(87)$ \\
& Uncomplicated & 14 & 28.5 & $8(57)$ & $6(43)$ \\
\hline
\end{tabular}

SAA $=$ serum amyloid A. a) Number and percentages of patients with SAA above and below the estimated clinical decision limit of SAA. b) Complicated neoplastic disorders were defined as known metastases, disseminated intravascular coagulation or lethargy. c) Complicated gastrointestinal disorders were defined as hemorrhagic gastroenteritis or gastrointestinal foreign bodies, compared to local, selflimiting gastrointestinal symptoms. 
SAA could not be used to differentiate between dogs with benign and malignant neoplasms (Fig. 2, Table 7). However, increased concentrations of SAA were observed in $68 \%$ (15 out of 22) of the dogs with systemic complications due to the neoplastic disorder such as known metastases, cachexia, DIC or lethargy, while only 3\% (2 out of 63) of neoplastic patients without such complications had a concentration of SAA exceeding the decision limit. SAA concentrations exceeding the decision limit were observed in 2 dogs with neoplastic diseases without complications. Both dogs were diagnosed, however, with malignant neoplasms, and incipient complications such as micro-metastases not detected by diagnostic imaging can consequently not be ruled out entirely.

A significantly larger proportion (13 out of 15) of dogs with hemorrhagic gastroenteritis or gastrointestinal foreign bodies had SAA concentrations exceeding the clinical decision limit compared to dogs with non-complicated gastrointestinal disorders (Fig. 2, Table 7). However, almost half of the patients with non-complicated gastroenteritis had SAA concentrations exceeding the clinical decision limit, and 2 out of 15 dogs had SAA concentrations below the clinical decision limit despite hemorrhagic gastroenteritis.

\section{DISCUSSION}

A clinically useful diagnostic performance of canine SAA measured by LAT was demonstrated in this study, which included dogs of a wide range of ages, breeds, and disorders, representative for a general clinical setting. The LAT is based on human monoclonal antibodies [7], eliminating the potential batch-to-batch variations of polyclonal based assays [16]. The automated measuring time of 10-15 min and random-access facilitate quick determinations of SAA for real-time diagnostic and monitoring purposes, more useful for routine use than the time-consuming methods traditionally used for measurements of canine SAA (e.g. ELISA) [22, 27, 28].

SAA in dogs with and without systemic inflammation: The significant overlap performance of SAA demonstrated in the study confirms the results of previous studies of canine SAA $[1,3,4,8]$, and extends the initial knowledge of the overlap performance of the LAT obtained as part of our validation of the assay [7]. Further, our comparison of the diagnostic performance of SAA with the performance of leukocytes, segmented and band neutrophils and body temperature suggests that routine measurements of SAA can be a useful adjunct to the information obtained by measuring these traditionally used inflammatory markers. Similar results have been reported in previous studies on SAA in other species $[12,19]$, but to the authors' knowledge, this is the first study documenting SAA to be a more sensitive and specific diagnostic marker in dogs compared to these more traditionally used parameters. The ROC analysis indicated an overall superiority in the whole spectrum of measurements independent of the chosen clinical decision limits [10]. The high diagnostic specificity is especially important in the comparison of SAA to the other diagnostic markers. Body

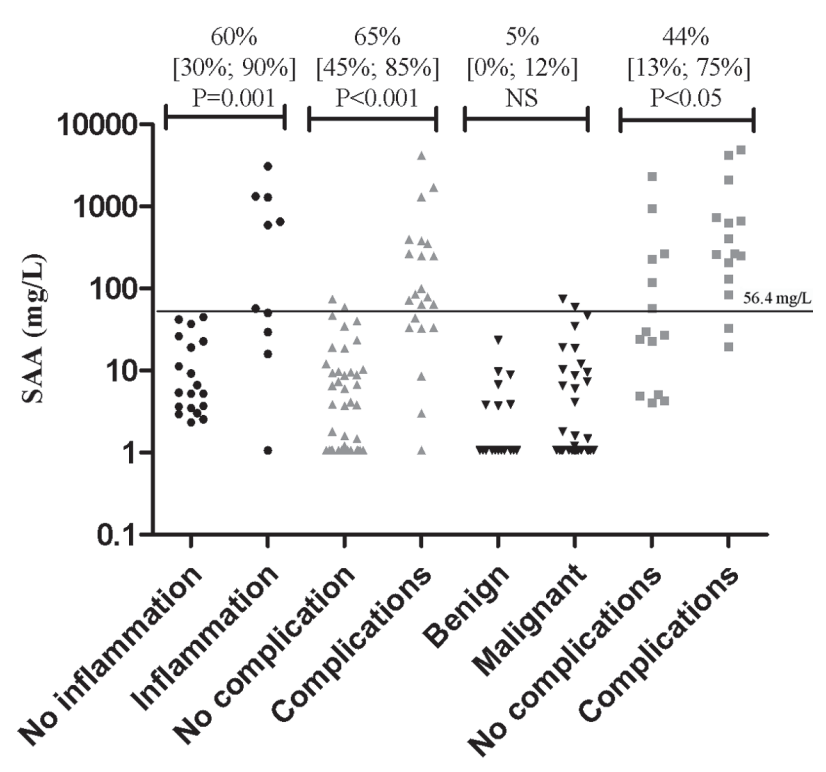

Fig. 2. Serum amyloid A (SAA) measurements in dogs with neurological disorders with or without inflammatory changes in cerebrospinal fluid and/or magnetic resonance imaging (black circles), in dogs with neoplasms with or without complications (known metastases, disseminated intravascular coagulation, cachexia, or lethargy) (grey triangles), in dogs with malignant and benign neoplasms (black triangles) and in dogs with gastrointestinal disorders with or without complications (hemorrhagic gastroenteritis or gastrointestinal foreign bodies) (grey squares). Chi-square tests were used to test for significant differences in proportions $(P<0.05)$, and differences in proportions of dogs with SAA concentrations above or below a clinical decision limit of $56.4 \mathrm{mg} / \mathrm{l}$ (horizontal line) are given in percentages [95\% confidence intervals]. Measurements below the detection limit were plotted as $1.06 \mathrm{mg} / l$ which is the previously calculated detection limit of the assay [7]. NS: Not significant.

temperature, leukocyte, and neutrophil counts are all known to be stress labile parameters decreasing the specificity of these parameters and increasing the clinical usefulness of an additional diagnostic marker, which can detect systemic inflammation with increased diagnostic specificity. In order for SAA to be used as a routine marker, knowledge about its clinical decision limit is needed, and useful clinical decision limits of SAA, measured by LAT, were consequently established in the study. However, it is important to note that exact clinical decision limits should be estimated locally [6], with ROC analysis as a useful method as demonstrated.

A previous study has reported that local inflammation as a result of dental disorders will not necessarily be accompanied by a systemic inflammatory reaction [2]. In our study, dogs with dental calculi were subsequently included in the group of diseased dogs without systemic inflammatory disorders. No dog in this group had SAA levels exceeding the established clinical decision limit, which confirms the results obtained previously [2]. The concentration of SAA increases when locally produced cytokines are transported to the liver resulting in a systemic inflammatory reaction [21]. Conse- 
quently, it can be expected that the concentration of SAAdoes not increase as long as the inflammation remains contained and localized, similarly to what is observed in dental patients [2]. In our study, patients have, therefore, been included in the group of diseased dogs without systemic inflammatory disorders, despite the presence of local inflammatory conditions such as cystitis, anal sacculitis, and chronic dermatitis, if no clinical signs of systemic complications were observed. All dogs in this group had concentrations of SAA below the clinical decision limit, indicating that serum SAA is a useful marker of systemic inflammation only.

SAA in dogs with diseases including varying degrees of systemic inflammation: Significant higher concentrations of SAA were observed in dogs with complicated neurologic, neoplastic and gastrointestinal disorders, compared to dogs with no systemic affection. The findings in neurological patients support the results of a previous study reporting increased concentrations of SAA in dogs with steroid-responsive meningitis [18], and consequently SAA seems like a promising parameter in the diagnostic work-up of neurological patients. However, increased concentrations of SAA were not detected in all dogs, despite inflammatory findings in CSF and/or MRI. Additional studies are necessary to explain these findings in detail, but a possible explanation could be that dogs with inflammatory neurological diseases are sometimes only affected locally [24], while SAA is only expected to increase in cases with systemic inflammation [21]. Further, a clear distinction between disorders with and without inflammation is not always possible in dogs with neurological symptoms despite a thorough diagnostic workup [25], and consequently, some patients with inconclusive classification can be expected.

The present study supports the findings previously demonstrated in a study of dogs with mammary gland neoplasms. In this study, dogs with metastases, large primary tumours and ulceration or secondary inflammation of the tumour tended to have higher concentrations of SAA than dogs with mammary neoplasms not complicated by such factors [23]. Similar findings have been observed in some human cancers, and SAA has been demonstrated to be a useful prognostic marker for the severity and clinical outcome in humans with certain types of cancers [5]. Further studies are needed in order to investigate the prognostic potential in measurements of SAA in canine neoplastic patients, but the results from our study indicate potential for the detection of a systemic inflammatory affection resulting from neoplasia.

A previous study has demonstrated increases in the concentration of SAA in dogs as a result of experimentally induced gastric mucosal injuries [1], and the present study suggests that SAA is a useful marker of the systemic inflammatory component of spontaneous gastrointestinal disorders as well. The present results of canine SAA correspond to a previous study of porcine SAA demonstrating significant higher concentrations of SAA in cases of hemorrhagic swine dysentery, compared to non-hemorrhagic cases [13]. Further studies including more and larger groups of dogs will be necessary in order to investigate the full diagnostic and prognostic potential of SAA as a clinical chemical parameter in canine gastrointestinal disorders, but some potential can be expected based on the results of our study.

The different conditions for storing and transport of samples can have affected the results obtained in the study, but additional studies are needed to investigate the exact influence of such factors.

In conclusion, SAA measured by the LAT can be used to distinguish groups of dogs with systemic inflammation from groups of dogs without a systemic inflammatory disorder by means of an established clinical decision limit. SAA was observed to be a significantly more accurate marker of systemic inflammation compared to the traditionally used inflammatory markers, body temperature, leukocyte and neutrophil counts, and can also be used to differentiate dogs with neurological, neoplastic, and gastrointestinal disorders with varying degrees of systemic affection. Consequently, the automated LAT makes SAA applicable for routine use in a general clinical setting.

ACKNOWLEDGMENTS. The study was supported by the EIKEN Chemical Co., Tokyo, Japan without any conflict of interest. The authors would like to thank the staff at the Central Laboratory, Department of Veterinary Clinical and Animal Sciences, University of Copenhagen for technical support during the progress of the study and to all the people, who have helped in the collection of samples to the study, including Professor MA Oyama, DACVIM-cardiology, and Professor LG King, DACVECC, and their staffs at the Department of Clinical Studies, School of Veterinary Medicine, University of Pennsylvania, U.S.A., and the staffs at: San Marco Veterinary Clinic, Padova, Italy; Blue Star Animal Hospital, Gothenburg, Sweden; the Department of Companion Animal Clinical Studies, University of Pretoria, South Africa; the Department of Clinical Sciences of Companion Animals, University of Utrecht, Netherlands, the Department of Animal Medicine and Surgery, University of Murcia, Spain; and the Department of Veterinary Clinical and Animal Sciences, University of Copenhagen, Denmark.

\section{REFERENCES}

1. Bayramli, G. and Ulutas, B. 2008. Acute phase protein response in dogs with experimentally induced gastric mucosal injury. Vet. Clin. Pathol. 37: 312-316. [Medline] [CrossRef]

2. Buttke, T. M., Shipper, G., Delano, E. and Trope, M. 2005. $\mathrm{C}$-reactive protein and serum amyloid $\mathrm{A}$ in a canine model of chronic apical periodontitis. J. Endod. 31: 728-732. [Medline] [CrossRef]

3. Ceron, J. J., Eckersall, P. and Martinez-Subiela, S. 2005. Acute phase proteins in dogs and cats: current knowledge and future perspectives. Vet. Clin. Pathol. 34: 85-99. [Medline] [CrossRef]

4. Chikamune, T., Katamoto, H., Nomura, K. and Ohashi, F. 1998. Lipoprotein profile in canine pancreatitis induced with oleic acid. J. Vet. Med. Sci. 60: 413-421. [Medline] [CrossRef]

5. Cho, W. C. S., Yip, T. T., Cheng, W. W. and Au, J. S. K. 2010. Serum amyloid A is elevated in the serum of lung cancer patients with poor prognosis. Br. J. Cancer 102: 1731-1735. [Medline] [CrossRef]

6. Christensen, M. B., Ceron, J. J., Tvarijonaviciute, A. and 
Kjelgaard-Hansen, M. 2012. Diagnostic measurements of canine serum amyloid A using automated latex agglutination turbidimetry: an interlaboratorial comparison. p. 155. In: Proceedings of 14th Conference of the European Society of Veterinary Clinical Pathology.

7. Christensen, M., Jacobsen, S., Ichiyanagi, T. and KjelgaardHansen, M. 2012. Evaluation of an automated assay based on monoclonal anti-human serum amyloid A (SAA) antibodies for measurement of canine, feline, and equine SAA. Vet. J. 194: 332-337. [Medline] [CrossRef]

8. Dąbrowski, R., Kostro, K., Lisiecka, U., Szczubiał, M. and Krakowski, L. 2009. Usefulness of C-reactive protein, serum amyloid A component, and haptoglobin determinations in bitches with pyometra for monitoring early post-ovariohysterectomy complications. Theriogenology 72: 471-476. [Medline] [CrossRef]

9. Elwood, C., Devauchelle, P., Elliott, J., Freiche, V., German, A. J., Gualtieri, M., Hall, E., den Hertog, E., Neiger, R., Peeters, D., Roura, X. and Savary-Bataille, K. 2010. Emesis in dogs: a review. J. Small Anim. Pract. 51: 4-22. [Medline] [CrossRef]

10. Gardner, I. A. and Greiner, M. 2006. Receiver-operating characteristic curves and likelihood ratios: improvements over traditional methods for the evaluation and application of veterinary pathology tests. Vet. Clin. Pathol. 35: 8-17. [Medline] [CrossRef]

11. Hall, E. J. 1999. Clinical laboratory evaluation of small intestinal function. Vet. Clin. North Am. Small Anim. Pract. 29: 441-469. [Medline]

12. Horadagoda, N. U., Knox, K., Gibbs, H., Reid, S., Horadagoda, A., Edwards, S. and Eckersall, P. 1999. Acute phase proteins in cattle: discrimination between acute and chronic inflammation. Vet. Rec. 144: 437-441. [Medline] [CrossRef]

13. Jacobson, M., Fellstrom, C., Lindberg, R., Wallgren, P. and Jensen-Waern, M. 2004. Experimental swine dysentery: comparison between infection models. J. Med. Microbiol. 53: 273-280. [Medline] [CrossRef]

14. Jensen, A. L., Bomholt, M. and Moe, L. 2001. Preliminary evaluation of a particle-enhanced turbidimetric immunoassay (PETIA) for the determination of serum cystatin C-like immunoreactivity in dogs. Vet. Clin. Pathol. 30: 86-90. [Medline] [CrossRef]

15. Jergens, A. E., Schreiner, C., Frank, D., Niyo, Y., Ahrens, F., Eckersall, P., Benson, T. and Evans, R. 2003. A scoring index for disease activity in canine inflammatory bowel disease. J. Vet. Intern. Med. 17: 291-297. [Medline] [CrossRef]

16. Kjelgaard-Hansen, M. 2010. Comments on measurement of Creactive protein in dogs. Vet. Clin. Pathol. 39: 402-403. [Medline] [CrossRef]

17. Kjelgaard-Hansen, M. and Jacobsen, S. 2011. Assay validation and diagnostic applications of major acute-phase protein testing in companion animals. Clin. Lab. Med. 31: 51-70. [Medline] [CrossRef]

18. Lowrie, M., Penderis, J., Eckersall, P. D., McLaughlin, M., Mellor, D. and Anderson, T. J. 2009. The role of acute phase proteins in diagnosis and management of steroid-responsive meningitis arteritis in dogs. Vet. J. 182: 125-130. [Medline] [CrossRef]

19. Mozes, G., Friedman, N. and Shainkinkestenbaum, R. 1989. Serum amyloid a: an extremely sensitive marker for intensity of tissue-damage in trauma patients and indicator of acute response in various diseases. J. Trauma 29: 71-74. [Medline] [CrossRef]

20. Peeters, M. E. and Kirpensteijn, J. 2011. Comparison of surgical variables and short-term postoperative complications in healthy dogs undergoing ovariohysterectomy or ovariectomy. J. Am. Vet. Med. Assoc. 238: 189-194. [Medline] [CrossRef]

21. Petersen, H. H., Nielsen, J. and Heegaard, P. 2004. Application of acute phase protein measurements in veterinary clinical chemistry. Vet. Res. 35: 163-187. [Medline] [CrossRef]

22. Tagata, K., Yokoyama, S., Ginbo, T., Honda, M., Okimura, T., Odakura, M., Nomura, M. and Yamamoto, S. 1996. Quantitative capillary reversed passive latex agglutination test for C-reactive protein (CRP) in the dog. Vet. Res. Commun. 20: 21-30. [Medline] [CrossRef]

23. Tecles, F., Caldin, M., Zanella, A., Membiela, F., Tvarijonaviciute, A., Martinez Subiela, S. and Ceron, J. 2009. Serum acute phase protein concentrations in female dogs with mammary tumors. J. Vet. Diagn. Invest. 21: 214-219. [Medline] [CrossRef]

24. Thomas, W. B. 1998. Inflammatory diseases of the central nervous system in dogs. Clin. Tech. Small Anim. Pract. 13: 167-178. [Medline] [CrossRef]

25. Tipold, A. 1995. Diagnosis of inflammatory and infectiousdiseases of the central-nervous-system in dogs: a retrospective study. J. Vet. Intern. Med. 9: 304-314. [Medline] [CrossRef]

26. Tvarijonaviciute, A., Martinez-Subiela, S., Carrillo-Sanchez, J. D., Tecles, F. and Ceron, J. J. 2011. Effects of orchidectomy in selective biochemical analytes in beagle dogs. Reprod. Domest. Anim. 46: 957-963. [Medline] [CrossRef]

27. Yamamoto, S., Shida, T., Okimura, T., Otabe, K., Honda, M., Ashida, Y., Furukawa, E., Sarikaputi, M. and Naiki, M. 1994. Determination of C-reactive protein in serum and plasma from healthy dogs and dogs with pneumonia by ELISA and slide reversed passive latex agglutination-test. Vet. Q. 16: 74-77. [Medline] [CrossRef]

28. Yamamoto, S., Tagata, K., Ishikawa, Y., Santsuka, H., Yamada, M., Morimatsu, M. and Naiki, M. 1993. Avidity of antibody and agglutinability of antibody-sensitized latex in latex agglutination-test. Vet. Immunol. Immunopathol. 36: 257-264. [Medline] [CrossRef] 